\title{
SU(3) Symmetry and Scissors Mode Vibrations in Nuclei
}

\author{
Yang Sun ${ }^{(1,2,3)}$, Cheng-Li Wu ${ }^{(4)}$, Kumar Bhatt ${ }^{(5)}$, Mike Guidry ${ }^{(1)}$ \\ (1) Department of Physics and Astronomy, University of Tennessee \\ Knoxville, Tennessee 37996, USA \\ ${ }^{(2)}$ Department of Physics, Tsinghua University, Beijing 100084, P. R. China \\ (3) Department of Physics, Xuzhou Normal University \\ Xuzhou, Jiangsu 221009, P. R. China \\ (4) National Center for Theoretical Science, Hsinchu, Taiwan 300, ROC \\ (5) Department of Physics and Astronomy, University of Mississippi \\ University, Mississippi 38677, USA
}

\begin{abstract}
We show that a nearly perfect $S U(3)$ symmetry emerges from an extended Projected Shell Model. Starting from a deformed potential we construct separate bases for neutron and proton collective rotational states by exact angular momentum projection. These rotational states are then coupled by diagonalizing a residual pairing plus quadrupole interaction. The states obtained exhibit a one-to-one correspondence with an $S U(3)$ spectrum up to high angular momentum and excitation, and their wave functions have a near-maximal overlap with the $S U(3)$ states. They can also be classified as rotational bands built on spin- $1 \hbar$ phonon excitations, which correspond to a geometrical scissors mode and its generalizations. This work is a direct demonstration that numerical angular momentum projection theory extends the Elliott's original idea to heavy nuclear systems.
\end{abstract}

\section{Introduction}

In the long history of the shell model, Elliott was the first to point out the advantage of using a deformed (intrinsic) many-body basis and developed the $S U(3)$ Shell Model [1] for $s d$-shell nuclei. In this model, the classification of basis states and their projection onto good angular momentum can be carried out using group theory. It works nicely as long as the spin-orbit force is weak. In heavier nuclei, where the presence of a strong spin-orbit force is essential for the correct shell closures, the high- $j$ orbital in an $N$-shell is strongly pushed 
down by this interaction and intrudes into a lower $N$-shell. This results in a re-classification of basis states and the Elliott $S U(3)$ scheme is no longer valid for heavy nuclei.

However, in early work of Bhatt, Parikh and McGrory [2], it was shown that wave functions for yrast states of deformed nuclei in the $1 f-2 p$ shell that were obtained in shell model calculations within a severely truncated configuration space that cannot support microscopic $S U(3)$ symmetry of Elliott, have $S U(3)$-like structures when the wave function of a state with total angular momentum $I$ is expressed in terms of coupling of the collective neutron and proton angular momenta $I_{\nu}$ and $I_{\pi}$ within the truncated configuration space. On the basis of this analysis, they suggested that the rotational features of yrast states in heavy, deformed nuclei could be due to a similar manifestation of $S U(3)$ symmetry occurring at the macroscopic level, even if it is absent at the single particle level in the usual basis. Brink et al. [3] suggested an alternative derivation without the use of symmetry arguments as well as the assumption of the motion of a particle in a static deformed field. Instead they approached the problem by diagonalising a rotationally invariant Hamiltonian using basis states of good angular momentum at all stages, which leads to the standard 'symmetrised' wavefunctions.

In our recent letter [4], the connection between the numerically calculated states of heavy nuclear system and such macroscopic $S U(3)$ symmetry states has been explored. The work was based on an extended Projected Shell Model (PSM). Starting from a deformed potential, instead of employing only one BCS vacuum as in the original Projected Shell Model [5], we use independent BCS condensates for neutrons and protons to construct separate bases for neutron and proton collective rotational states by exact angular momentum projection. The active nucleons in the model space were permitted to occupy three full major shells, which is a space far larger than a conventional shell model can handle. These rotational states were then coupled by diagonalizing a residual pairing plus quadrupole interaction. Many new bands emerge that are not contained in the original PSM, and it was shown that these bands exhibit a nearly perfect $S U(3)$ spectrum, even though there is no explicit dynamical symmetry in such a model.

Our work [4] demonstrates a clear connection between a numerical microscopic method, which starts from certain set of intrinsic states, and an elegant group theoretical description of nuclear states. Thus the PSM may be viewed as a natural method to investigate the emergence of $S U(3)$ symmetry as a truly dynamical symmetry in heavy, deformed nuclear systems.

The present paper elaborates on our previous letter [4]. The paper is organized as follows: In Section 2, we introduce the extended PSM with an emphasis on how to treat coupled rotations of neutron and proton systems. It is shown that 
this treatment generalizes the original PSM by allowing for relative motion between the neutron and proton systems. In Section 3, we give details of the calculation for an example, ${ }^{168} \mathrm{Er}$. A much richer spectrum is obtained after diagonalization than from the usual PSM. With the help of group theory, the states obtained from the diagonalization are shown to possess a strong $S U(3)$ symmetry. We further demonstrate that these states can be classified as rotational bands built on spin- $1 \hbar$ phonon excitations, and we suggest that these correspond to a geometrical scissors mode and its generalizations. These states are interpreted in Section 4. In Section 5, we buttress our conclusions by comparing the angular momentum distribution in our deformed basis states and the intrinsic states of the $S U(3)$ model, and by evaluating overlaps of our total wave functions with the $S U(3)$ ones. Then we calculate $B(E 2)$ values from the PSM wave functions to further confirm the state classifications. Some comments about the emergent $S U(3)$ symmetry and about a possible relation to scissors mode vibrations are made in Section 6 and 7, respectively. The lowest excitation bands, the first $1^{+}$and the first $2^{+}$band, as well as the second $0^{+}$band, are further discussed in Section 8 . Finally, we summarize the paper in Section 9.

\section{Extension of the Projected Shell Model}

The shell model is the most fundamental way of describing many-nucleon systems fully quantum mechanically. However, using the shell model to study deformed heavy nuclei is a desirable but very difficult task because of large dimensionality and its related problems. Even with today's computer power, the best standard shell model diagonalization can be done only in the full $p f$ shell space, as for example in the work of the Strasbourg-Madrid group [6], for which the dimension of the configuration space is well over one million. The PSM provides one possible solution for this difficulty. In this approach, one first truncates the configuration space with guidance from the deformed mean field by selecting only the BCS vacuum plus a few quasiparticle configurations in the Nilsson orbitals around the Fermi surface, performs angular momentum projection (and particle number projection if required) to obtain a set of laboratory-frame basis states, and finally diagonalizes a shell-model Hamiltonian in this space. Since the deformed mean field + BCS vacuum already incorporates strong particle-hole and particle-particle correlations, this truncation should be appropriate for the low-lying states dominated by quadrupole and pairing collectivity. Indeed, this approach has been very successful for ground band (g-band) properties and near-yrast quasiparticle excitations in high-spin physics for both normally deformed and superdeformed states [5,7-9].

However, in this formalism the basis vacuum is the usual BCS condensate of neutrons and protons in a fixed deformed potential (fixed shape in the 
geometric models). Without quasiparticle excitations, one obtains only the gband after angular momentum projection. Thus, there is no room for studying any other collective excitations.

It is known that small perturbations of nuclear shapes and relative orientations around the equilibrium can give rise to physical states at low to moderate excitation energies. Classical examples of such motion include $\beta$ - and $\gamma$-vibrations [10], in which neutrons and protons undergo vibrations as a collective system. These small-amplitude motions are not built into the ground state for theories like Hartree-Fock-Bogoliubov or BCS. One may obtain the $\beta$ - and $\gamma$-vibrations by building additional correlations into the ground state, as for example in the Random Phase Approximation (see, for example, Ref. [11]), or by enriching the intrinsic degrees of freedom in the deformed potential [12].

Another possible mode of collective motion results if we consider neutron and proton fields of the fixed deformation, but permit small oscillations in the relative orientations of these fields $[13,14]$. This geometric picture may be related to the two-rotor model [15]. Because of the strong restoring force [16] (with its physical origin in the neutron-proton interaction), this oscillation is confined to a small angle between the protons and neutrons (thus it is termed small-amplitude scissors motion). This vibration, and the $\beta$ - and $\gamma$-vibrations, can be classified using group theoretical methods; they belong to the lowest collective excitations of the ground state, as pointed out by Iachello [17].

As introduced in our previous letter [4], in order to study microscopically the relative motion between neutron and proton intrinsic states with a fixed deformation, we have extended the original PSM in the following manner: Instead of a single BCS vacuum, the angular momentum projection is now performed for separate neutron and proton deformed BCS vacua. Although the introduction of two separately projected BCS vacua seems to treat neutrons and protons as two independent systems, the equal deformation used in the Nilsson calculation of the basis already contains strong correlation between the two systems [18]. Further proton-neutron correlations are introduced by explicitly diagonalizing the total Hamiltonian in the basis formed by the angular momentum projected neutron and proton states. This procedure gives the usual g-band corresponding to the coherently coupled BCS condensate of neutrons and protons, but also leads to a new set of states built on a more complex vacuum that incorporates fluctuations in the relative orientation of the neutron and proton fields of constant deformation.

The neutron and proton valence spaces employed in the present work are those of Ref. [5]. Our single particle space contains three major shells $(N=4,5$, and 6$)$ for neutrons and ( $N=3,4$ and 5$)$ for protons; this has been shown to be sufficient for a quantitative description of rare-earth g-bands and bands built on a few quasiparticle excitations [5]. Separable forces of pairing plus 
quadrupole type [19] are used in our Hamiltonian. We note that a recent study by Dufour and Zuker [20] has shown explicitly that the residual part of the realistic force is strongly dominated by pairing and quadrupole interactions.

The Hamiltonian [5] can be expressed as $\hat{H}=\hat{H}_{\nu}+\hat{H}_{\pi}+\hat{H}_{\nu \pi}$, where $H_{\tau}(\tau=$ $\nu, \pi)$ is the like-particle pairing plus quadrupole Hamiltonian, with inclusion of quadrupole-pairing,

$$
\hat{H}_{\tau}=\hat{H}_{\tau}^{0}-\frac{\chi_{\tau \tau}}{2} \sum_{\mu} \hat{Q}_{\tau}^{\dagger \mu} \hat{Q}_{\tau}^{\mu}-G_{\mathrm{M}}^{\tau} \hat{P}_{\tau}^{\dagger} \hat{P}_{\tau}-G_{\mathrm{Q}}^{\tau} \sum_{\mu} \hat{P}_{\tau}^{\dagger \mu} \hat{P}_{\tau}^{\mu}
$$

and $\hat{H}_{\nu \pi}$ is the $\mathrm{n}-\mathrm{p}$ quadrupole-quadrupole residual interaction

$$
\hat{H}_{\nu \pi}=-\chi_{\nu \pi} \sum_{\mu} \hat{Q}_{\nu}^{\dagger \mu} \hat{Q}_{\pi}^{\mu}
$$

In Eq. (1), the four terms are, respectively, the spherical single-particle energy which contains a proper spin-orbit force [21], the quadrupole-quadrupole interaction, the monopole-pairing, and the quadrupole-pairing interaction. The interaction strengths $\chi_{\tau \tau}(\tau=\nu$ or $\pi)$ are related self-consistently to the deformation $\varepsilon$ by $[5]$

$$
\chi_{\tau \tau}=\frac{\frac{2}{3} \varepsilon\left(\hbar \omega_{\tau}\right)^{2}}{\hbar \omega_{\nu}\left\langle\hat{Q}_{0}\right\rangle_{\nu}+\hbar \omega_{\pi}\left\langle\hat{Q}_{0}\right\rangle_{\pi}} .
$$

Obviously, neutrons and protons are coupled by the self-consistency condition. Following Ref. [5], the strength $\chi_{\nu \pi}$ is assumed to be $\chi_{\nu \pi}=\left(\chi_{\nu \nu} \chi_{\pi \pi}\right)^{1 / 2}$. Similar parameterizations were used in earlier work [19].

In this paper, we concentrate on collective motion only and do not consider quasiparticle excitations; however, we shall discuss possible physical implication of including quasiparticle states in a later section. Particle number projection is not included, so particle number is conserved only on average in the BCS calculation. It has been shown by a systematic calculation [5] that particle number projection does not alter the unprojected results very much for those well-deformed heavy nuclei. We do not expect that particle number projection will change conclusions of this paper with respect to the emergence of $S U(3)$ symmetry.

The intrinsic state $|0\rangle$ of an axially deformed nucleus is, in this approximation, taken to be the product of the Nilsson-BCS quasiparticle vacuum states $\left|0_{\nu}\right\rangle$ and $\left|0_{\pi}\right\rangle$

$$
|0\rangle=\left|0_{\nu}\right\rangle\left|0_{\pi}\right\rangle
$$


The original PSM ground band with angular momentum $|I\rangle$ is obtained by angular momentum projection [5] onto the vacuum:

$$
|I\rangle=\aleph^{I} \hat{P}^{I}|0\rangle
$$

where $\hat{P}^{I}$ is the (one-dimensional) angular momentum projection operator [22]

$$
\hat{P}^{I}=\left(I+\frac{1}{2}\right) \int_{0}^{\pi} d \beta \sin \beta d^{I}(\beta) \hat{R}(\beta),
$$

with $d^{I}(\beta)$ the small $d$-function and $\hat{R}(\beta)$ the one-dimensional rotational operator $[23]$ and $\aleph^{I}$ is the normalization constant,

$$
\aleph^{I}=\left\langle 0\left|\hat{P}^{I}\right| 0\right\rangle^{-\frac{1}{2}}
$$

In the extended PSM, we first project out the neutron (proton) states $\left|I_{\nu}\right\rangle$ $\left(\left|I_{\pi}\right\rangle\right)$ with angular momentum $I_{\nu}\left(I_{\pi}\right)$ from the intrinsic state $\left|0_{\nu}\right\rangle\left(\left|0_{\pi}\right\rangle\right)$. The projected states $\left|I_{\nu}\right\rangle$ and $\left|I_{\pi}\right\rangle$ are coupled to form the basis states $\left|\left(I_{\nu} \otimes I_{\pi}\right) I\right\rangle$ for different total angular momentum $I$. These basis states are used to construct the matrix of the total Hamiltonian of Eqs. (1) and (2),

$$
\begin{aligned}
\left\langle\left(I_{\nu} \otimes I_{\pi}\right) I\right| & \hat{H}\left|\left(I_{\nu}^{\prime} \otimes I_{\pi}^{\prime}\right) I\right\rangle \\
= & {\left[\left\langle I_{\nu}\left|\hat{H}_{\nu}\right| I_{\nu}^{\prime}\right\rangle+\left\langle I_{\pi}\left|\hat{H}_{\pi}\right| I_{\pi}^{\prime}\right\rangle\right] \delta_{I_{\nu} I_{\nu}^{\prime}} \delta_{I_{\pi} I_{\pi}^{\prime}} } \\
& -\chi_{\nu \pi}\left\langle\left(I_{\nu} \otimes I_{\pi}\right) I\left|\hat{Q}_{\nu}^{\dagger} \hat{Q}_{\pi}\right|\left(I_{\nu}^{\prime} \otimes I_{\pi}^{\prime}\right) I\right\rangle .
\end{aligned}
$$

The last term in Eq. (8) can be written explicitly as [10]

$$
\begin{aligned}
\left\langle\left( I_{\nu}\right.\right. & \left.\left.\otimes I_{\pi}\right) I\left|\hat{Q}_{\nu}^{\dagger} \hat{Q}_{\pi}\right|\left(I_{\nu}^{\prime} \otimes I_{\pi}^{\prime}\right) I\right\rangle \\
& =\mathcal{W}\left(I_{\pi} 2 I I_{\nu}^{\prime} ; I_{\pi}^{\prime} I_{\nu}\right)\left\langle I_{\nu}\left\|\hat{Q}_{\nu}\right\| I_{\nu}^{\prime}\right\rangle \\
& \times\left\langle I_{\pi}\left\|\hat{Q}_{\pi}\right\| I_{\pi}^{\prime}\right\rangle / \sqrt{\left(2 I_{\nu}+1\right)\left(2 I_{\pi}^{\prime}+1\right)},
\end{aligned}
$$

where $\mathcal{W}$ is the $6-j$ symbol.

The term $\left\langle I_{\nu}\left|\hat{H}_{\nu}\right| I_{\nu}\right\rangle\left(\left\langle I_{\pi}\left|\hat{H}_{\pi}\right| I_{\pi}\right\rangle\right)$ is the energy of the state $\left|I_{\nu}\right\rangle\left(\left|I_{\pi}\right\rangle\right)$ projected from the intrinsic state $\left|0_{\nu}\right\rangle\left(\left|0_{\pi}\right\rangle\right)$ with the neutron (proton) part of Hamiltonian $\hat{H}_{\nu}\left(\hat{H}_{\pi}\right)$ given by Eq. (1). The Hamiltonian matrix of Eq. (8) is diagonalized and the resulting PSM eigenstates $|\alpha, I\rangle_{\mathrm{PSM}}$ are expressed as a linear combination of the basis states $\left|\left(I_{\nu} \otimes I_{\pi}\right) I\right\rangle$ :

$$
|\alpha, I\rangle_{\mathrm{PSM}}=\sum_{I_{\nu} I_{\pi}} f_{\mathrm{PSM}}^{\alpha}\left(I_{\nu} I_{\pi} ; I\right)\left|\left(I_{\nu} \otimes I_{\pi}\right) I\right\rangle
$$


where $\alpha$ labels different eigenstates having the same angular momentum. We shall show in the following sections that the amplitudes $f_{\mathrm{PSM}}^{\alpha}\left(I_{\nu} I_{\pi} ; I\right)$ carry information about $S U(3)$ symmetry emerging dynamically from the extended PSM.

\section{An Example: The Spectrum of ${ }^{168} \mathbf{E r}$}

Let us take as a typical example the rotational nucleus, ${ }^{168} \mathrm{Er}$. The deformed basis is constructed at deformation $\varepsilon_{2}=0.273$ for this nucleus [24]. The numerical values of the parameters in the Hamiltonian (Eqs. (1) and (2)) appropriate for this nucleus are (in MeV): $\chi_{\nu \nu}=0.0206, \chi_{\pi \pi}=0.0160, \chi_{\nu \pi}=0.0182$, $G_{M}^{\nu}=0.1049$, and $G_{M}^{\pi}=0.1346$. The ratio of the strength of the quadrupolepairing interaction to that of the monopole-pairing interaction is 0.16. All these values are the same as those employed in the early paper of Hara and Iwasaki [25] and as those in the review article of Hara and Sun [5]. The states $\left|I_{\nu}\right\rangle\left(\left|I_{\pi}\right\rangle\right)$ with angular momenta $I_{\nu}=0,2, \ldots, 32\left(I_{\pi}=0,2, \ldots, 16\right)$ were projected from the Nilsson-BCS vacuum state $\left|0_{\nu}\right\rangle\left(\left|0_{\pi}\right\rangle\right)$. We have omitted in this calculation the neutron states with $I_{\nu}>32$ and the proton states with $I_{\pi}>16$ because their probabilities in the intrinsic states are very small $(<$ $0.001)$.

The energy spectra $E\left(I_{\tau}\right)=\left\langle I_{\tau}\left|\hat{H}_{\tau}\right| I_{\tau}\right\rangle$ obtained from the projected states $\left|I_{\tau}\right\rangle$ are almost rotational $\left(E\left(I_{\tau}\right)=A_{\tau} I_{\tau}\left(I_{\tau}+1\right)\right.$ ), with the moment of inertia parameters $A_{\nu}=0.019 \mathrm{MeV}$ and $A_{\pi}=0.048 \mathrm{MeV}$.

For each spin $I$, we diagonalize Eq. (8) in the basis $\left|\left(I_{\nu} \otimes I_{\pi}\right) I\right\rangle$. The resulting spectrum with energy up to $20 \mathrm{MeV}$ and angular momenta up to $12 \hbar$ is shown in Fig. 1. It shows strikingly regular structure.

The lowest band is the g-band, which is nearly identical to that of earlier PSM calculations [26] with the same Hamiltonian where no separation and recoupling of neutron and proton components was considered. The calculated g-band in Fig. 1 reproduces the experimental g-band [27] very well, as we shall show later in Fig. 4. In addition to the g-band, many new excited bands emerge that are not found in the earlier PSM calculations. These bands exhibit a curvature similar to the g-band, suggesting that their moments of inertia are similar to the g-band. 


\section{Emergence of SU(3) Symmetry}

The strikingly regular pattern shown in Fig. 1 can be understood as manifestation of a nearly perfect $S U(3)$ symmetry: all bands can be well classified as a spectrum with $S U(3)$ symmetry if the projected neutron and proton BCS vacuum states are considered to be two independent $S U(3)$ representations coupled through the $Q_{\nu}-Q_{\pi}$ interaction.

This may be demonstrated by considering a model Hamiltonian with $S U(3)^{\nu} \otimes$ $S U(3)^{\pi} \supset S U(3)^{\nu+\pi}$ dynamical symmetry expressed in the form (see Eq. (3.107) in Ref. [28]):

$$
\hat{H}=\chi_{\nu}^{\mathrm{eff}} \hat{C}_{s u 3}^{\nu}+\chi_{\pi}^{\mathrm{eff}} \hat{C}_{s u 3}^{\pi}-\chi_{\nu \pi}^{\mathrm{eff}} \hat{C}_{s u 3}^{\nu+\pi}+A \hat{I}^{2},
$$

where $\hat{C}_{\text {su3 }}^{\tau}$ are the $S U(3)^{\tau}(\tau=\nu, \pi)$ Casimir operators for neutrons, protons, and the $\mathrm{n}-\mathrm{p}$ coupled symmetry $S U(3)^{\nu+\pi}(\tau=\nu+\pi)$. The eigenvalue of the lowest-order $S U(3)$ Casimir operator for a given representation $(\lambda, \mu)$ is $C(\lambda, \mu)=\frac{1}{2}\left(\lambda^{2}+\mu^{2}+\lambda \mu+3 \lambda+3 \mu\right)$. We assume that the two BCS vacua correspond to the $S U(3)$ symmetric representations $\left(\lambda_{\nu}, 0\right)$ and $\left(\lambda_{\pi}, 0\right)$, respectively, and that the permissible irreps $(\lambda, \mu)$ of $S U(3)^{\nu+\pi}$ are given by the Littlewood rule, $(\lambda, \mu)=\left(\lambda_{m}-2 \mu, \mu\right)$, where $\lambda_{m}=\lambda_{\nu}+\lambda_{\pi}$ is the maximum value of $\lambda$ and $\mu=0,1,2, \ldots, \lambda_{\pi}$, if $\lambda_{\pi} \leq \lambda_{\nu}$.

The eigenvalue spectrum $E[(\lambda, \mu) I]$ of the Hamiltonian of (11) obtained from the states with angular momentum $I$ belonging to the coupled representation $\left\{\left[\lambda_{\nu}, 0\right] \otimes\left[\lambda_{\pi}, 0\right]\right\}(\lambda, \mu) I$ is

$$
\begin{aligned}
E[(\lambda, \mu) I]-E_{\text {g.s. }} & =\chi_{\nu \pi}^{\mathrm{eff}}\left[C\left(\lambda_{m}, 0\right)-C\left(\lambda=\lambda_{m}-2 \mu, \mu\right)\right]+A I(I+1) \\
& =\mu \hbar \omega_{\infty}\left[1-\frac{\mu-1}{\lambda_{m}}\right]+A I(I+1),
\end{aligned}
$$

with

$$
\hbar \omega_{\infty}=\frac{3}{2} \lambda_{m} \chi_{\nu \pi}^{\mathrm{eff}}
$$

The second term in Eq. (12) gives a rotational band with a moment of inertia parameter $A=\hbar^{2} / 2 \Im$. $E_{\text {g.s. }}$ is the ground state energy corresponding to the coupled $S U(3)$ representation $\left[\lambda_{m}, 0\right]$

$$
E_{\text {g.s. }}=\chi_{\nu}^{\mathrm{eff}} C\left(\lambda_{\nu}, 0\right)+\chi_{\pi}^{\mathrm{eff}} C\left(\lambda_{\pi}, 0\right)-\chi_{\nu \pi}^{\mathrm{eff}} C\left(\lambda_{m}, 0\right) .
$$

The parameter $\lambda_{m}$ fixes the $S U(3)$ representation $\left[\lambda_{m}, 0\right]$ of the g-band. The 
parameter $\mu$ then fixes the representation $\left[\lambda=\lambda_{m}-2 \mu, \mu\right]$ of the excited bands through the Littlewood rule.

A more physical meaning of the parameter $\mu$ emerges from the first term of Eq. (12), which represents a vibration-like spectrum which tends to be harmonic for $\lambda_{m} \gg \mu$. In this limit, $\mu$ can be interpreted as the number of phonons of this "vibration" and $\hbar \omega_{\infty}$ as the one-phonon excitation energy as $\lambda_{m} \rightarrow \infty$, with its value being, approximately, the energy of the first excited $I=1^{+}$state relative to the ground state. The allowed angular momenta $I$ belonging to a representation $[\lambda, \mu]$ are determined from the usual $S U(3)$ subgroup reduction rules [28]. For example, the representation $(\lambda, \mu)=(40,4)$ can have a $K=0$ band with $I=0,2,4, \ldots, 44$, a $K=2$ band with $I=2,3,4, \ldots, 43$ and a $K=4$ band with $I=4,5,6, \ldots, 41[29]$.

The PSM calculated spectrum shown in Fig. 1 can then be described by the $S U(3)$ symmetry spectrum of Eq. (12). The parameters $\lambda_{m}, \hbar \omega_{\infty}$ and $A$ of Eq. (12), which fit best the PSM spectrum of Fig. 1, are $\lambda_{m}=48, \hbar \omega_{\infty}=2.9$ $\mathrm{MeV}\left(\chi_{\nu \pi}^{\mathrm{eff}}=0.0403\right)$, and $A=0.013 \mathrm{MeV}$. The $S U(3)$ band structure obtained with these parameters is shown in Fig. 1 as the dashed lines and reproduces remarkably well the PSM spectrum obtained from numerical projection and diagonalization. The fact that the parameter $\lambda_{m}=48$ fits the spectrum in Fig. 1 suggests that the g-band of PSM may be associated with the $S U(3)$ representation $\left[\lambda_{m}, 0\right]=[48,0]$. All the states shown in Fig. 1 can be labeled by the $S U(3)$ irrep labels $(\lambda, \mu)$ and the bandhead of each rotational band within the irrep is labeled by the $S U(3)$ quantum number $K$. Degeneracy at each spin may be deduced by counting one for each $K$ band, except only even spins are present for $K=0$ bands. We list the degeneracy of the $(40,4)$ representation as an example in Fig. 1. Not all PSM states can be seen clearly in the plot because of the high degeneracy, but there is a one-to-one correspondence between all predicted $S U(3)$ states and those observed in the PSM calculation.

One can see from Eq. (12) and the $S U(3)$ reduction rules that the whole spectrum of Fig. 1 can be viewed as a set of rotational bands built on different multi-phonon excitation states with a phonon energy $\hbar \omega=\hbar \omega_{\infty}\left[1-\frac{\mu-1}{\lambda_{m}}\right]$ and phonon spin $1 \hbar$. For example, a 3-phonon system could have two allowed states with energy $3 \hbar \omega$ and total spin $1 \hbar$ and $3 \hbar$; a 4 -phonon system could have three states with energy $4 \hbar \omega$ and total spin $0 \hbar, 2 \hbar$, and $4 \hbar$; and so on. This provides an alternative explanation of the degeneracy of the bands obtained by the PSM diagonalization. Comparing the $S U(3)$ and phonon classifications, one can see clearly that the $S U(3)$ quantum numbers $\mu$ and $K$ in Fig. 1 may be interpreted as the number of phonons and their allowed total spins, respectively.

As long as $\hbar \omega_{\infty}$ is held constant, the spectrum is sensitive to the effective $S U(3)$ quantum number $\lambda_{m}$ only through a small anharmonicity. For the present example, the phonon energy decreases smoothly from $2.9 \mathrm{MeV}$ to 2.5 
$\mathrm{MeV}$ as the phonon number increases from 1 to 7 . If $\lambda_{m} \rightarrow \infty$, then $\hbar \omega \rightarrow \hbar \omega_{\infty}$ and the vibration becomes harmonic. Thus, the anharmonicity originates in the finite number of particles for the nuclear system.

\section{Further Inspection on the Wave Functions}

The remarkably quantitative agreement of all calculated excited states with the $S U(3)$ spectrum demonstrated above makes it essentially certain that an $S U(3)$ symmetry emerges from the purely numerical PSM calculation. To remove all doubt, we may study the wave functions. In this section, we shall do this in three steps: we first compare angular momentum components in the PSM and $S U(3)$ intrinsic states. Then, we directly calculate overlaps of the actual wave functions from the PSM calculation with $S U(3)$ wave functions. Finally, we compute $B(E 2)$ values from PSM calculations that connect states in each band and compare with those of the $S U(3)$ model.

\subsection{SU(3) Structure of the Intrinsic States}

Let us start by recalling that an intrinsic state $\left|0_{\tau}\right\rangle,(\tau=\nu, \pi)$, is related to a projected state $\left|I_{\tau}\right\rangle$ by

$$
\left|0_{\tau}\right\rangle=\sum_{I_{\tau}} C_{I_{\tau}}\left|I_{\tau}\right\rangle .
$$

In Eq. (15), $C_{I}$ is the norm matrix element in the PSM described in Eq. (2.33) of Ref. [5]

$$
\left|C_{I}\right|^{2}=\left\langle 0\left|\hat{P}^{I}\right| 0\right\rangle .
$$

It gives the probability of finding angular momentum $I$ in the intrinsic state $|0\rangle$ (with $\sum_{I}\left|C_{I}\right|^{2}=1$, a sum rule obtained in Eq. (A80) of Ref. [5]). In other words, the quantity $\left|C_{I}\right|^{2}$ describes the angular momentum distribution in the intrinsic state $|0\rangle$. For the present calculation, we projected the states with angular momenta $I_{\nu}=0,2, \ldots, 32$ and $I_{\pi}=0,2, \ldots, 16$ from the Nilsson-BCS intrinsic states $\left|0_{\tau}\right\rangle$ of ${ }^{168} \mathrm{Er}$. The probabilities $\left|C_{I_{\tau}}\right|^{2}$ for $I_{\nu}>32$ and $I_{\pi}>16$ were very small and hence such states were not included in the basis state $\left|\left[I_{\nu} \otimes I_{\pi}\right] I\right\rangle$ used in the present PSM diagonalization. The values $\left|C_{I_{\tau}}\right|^{2}$ (for the nucleus ${ }^{168} \mathrm{Er}$ ) calculated for the first nine values $I_{\tau}=0,2, \ldots, 16$ contained in the intrinsic state $\left|0_{\tau}\right\rangle$ are listed in Table 1. For comparison, the values $\left|C_{I}\right|^{2}$ for the state $|I\rangle$ in the total intrinsic vacuum state $\left|0_{\nu}\right\rangle\left|0_{\pi}\right\rangle$ are also presented in the same table. 
In order to make a connection with the emergent $S U(3)$ symmetry at the intrinsic state level, we compare the probabilities $C_{I_{\tau}}^{2}(\mathrm{PSM})$ of the PSM intrinsic states $\left|0_{\tau}\right\rangle_{\mathrm{PSM}}$ with the corresponding probabilities $C_{I_{\tau}}^{2}(S U(3))$ in the intrinsic states $\left|0_{\tau}\right\rangle_{S U(3)}$ belonging to axially symmetric $S U(3)$ representation $\left[\lambda_{\tau}, 0\right]$. The latter probabilities are given by Elliott's $a((\lambda, 0) I)$ coefficients $[1]$. Analytical formulas for the $a((\lambda, \mu) I)$ are given in Table 2A of the Vergados's paper [29]. The effective $S U(3)$ representation $\left[\lambda_{\tau}, 0\right]$ for protons and neutrons is determined by equating the probabilities $C_{I_{\tau}}^{2}(\mathrm{PSM})=C_{I_{\tau}}^{2}(S U(3))=$ $a\left(\left(\lambda_{\tau}, 0\right) I_{\tau}\right)$ for a given $I_{\tau}$ (say $\left.I_{\tau}=0\right)$. For our present case of ${ }^{168} \mathrm{Er}$, the values of $\lambda_{\tau}$ obtained by this procedure are $\lambda_{\nu} \approx 32$ and $\lambda_{\pi} \approx 16$, to the nearest even integer.

The coefficients $a\left(\left(\lambda_{\tau}, 0\right) I\right)$ are then calculated for all values of $I_{\tau}=0,2, \ldots, \lambda_{\tau}$, allowed by the effective $\lambda_{\tau}$. (We denote these values as $a_{I}$ in the following discussion.) The $a_{I_{\tau}}$ values are compared with the corresponding $C_{I_{\tau}}$ values of the PSM in Fig. 2. The excellent agreement between these values shows that the distribution of angular momenta of neutrons and protons in our NilssonBCS vacuum states are very similar to those in the intrinsic states of the $S U(3)$-representations $\left[\lambda_{\nu}=32,0\right]$ and $\left[\lambda_{\pi}=16,0\right]$.

The total Nilsson-BCS vacuum state $|0\rangle_{\mathrm{PSM}}=\left|0_{\pi}\right\rangle_{\mathrm{PSM}}\left|0_{\nu}\right\rangle_{\mathrm{PSM}}$ should then have an effective $S U(3)$-representation $\left[\lambda=\lambda_{\pi}+\lambda_{\nu}, 0\right]=[48,0]$ and the probabilities $C_{I}^{2}$ of finding angular momentum $I$ in the total PSM intrinsic state should be similar to the probabilities $a((\lambda=48,0) I)$ corresponding to the $S U(3)$ intrinsic state of representation [48,0]. The agreement between these two probabilities is also shown in Fig. 2.

These PSM effective $S U(3)$ representations for the Nilsson-BCS intrinsic states of neutrons and protons for ${ }^{168} \mathrm{Er}$ in the three-shell valence space should be compared with the effective representations $\left[\lambda_{\nu}^{\mathrm{eff}}, \mu_{\nu}^{\mathrm{eff}}\right]=[40,0],\left[\lambda_{\pi}^{\mathrm{eff}}, \mu_{\pi}^{\mathrm{eff}}\right]=$ $[24,0]$, and $\left[\lambda^{\mathrm{eff}}, \mu^{\mathrm{eff}}\right]=[64,0]$ obtained by Kahane et al. [30] for just the Nilsson intrinsic state of ${ }^{168} \mathrm{Er}$ in the $\nu(82-126)$ and $\pi(50-82)$ single major shell valence space. These representations are larger than the effective representations $\left[\lambda_{\nu}=32,0\right]$ and $\left[\lambda_{\pi}=16,0\right]$ mainly because Kahane et al. have included all the projected states with probabilities $\left|C_{I}\right|^{2}<0.001$, which have been omitted in the present PSM calculations. For comparison, we also note that in the one major shell valence space, the pseudo- $S U(3)$ representations for the normal parity nucleons in ${ }_{68}^{168} \operatorname{Er}_{100}$ are $[31]\left[\lambda_{\nu}^{\text {pseudo }}, \mu_{\nu}^{\text {pseudo }}\right]=[20,4]$, $\left[\lambda_{\pi}^{\text {pseudo }}, \mu_{\pi}^{\text {pseudo }}\right]=[10,4]$, and $\left[\lambda_{n}^{\text {pseudo }}=30, \mu_{n}^{\text {pseudo }}=8\right]$. These representations are strongly triaxial but their effective axially symmetric representations would be $\left[\lambda_{\nu}^{\mathrm{eff}}=24,0\right],\left[\lambda_{\pi}^{\mathrm{eff}}=14,0\right]$ and $\left[\lambda^{\mathrm{eff}}=38,0\right]$. In the FDSM [28], the $S U(3)$ representation for 10 neutrons in the normal parity states of the $(82-126)$ shell is $\left[\lambda_{\nu}^{\mathrm{FDSM}}, \mu_{\nu}^{\mathrm{FDSM}}\right]=[10,0]$. There are also 10 protons in normal parity states of the $(50-82)$ shell, and they do not show an $S U(3)$, but an $S O(6)$ symmetry that corresponds to a $\gamma$-soft rotor. However, when the protons are 
strongly coupled to the neutrons, they will be synchronized with neutrons and stabilized at $\gamma \approx 0$, so that the proton system behaves like an $S U(3)$ rotor. Therefore, an effective $S U(3)$ representation $\left[\lambda_{\pi}^{\mathrm{FDSM}}, \mu_{\pi}^{\mathrm{FDSM}}\right]=[10,0]$ could be assigned to the protons. Quantitatively, the FDSM with one major shell can only fit to the first three bands in Fig. 1. Larger deviations are expected for the higher bands, although the qualitative band structure remains correct. If the $S U(3)$ symmetry which appears to emerge from the PSM calculation presented here is identified as the FDSM symmetry in the extended three-shell valence space with the effective neutron and proton numbers $n_{\nu}^{\text {eff }}=32$ and $n_{\pi}^{\text {eff }}=16$, then agreement with the PSM results is perfect, as shown in Fig. 1.

\subsection{SU(3) Symmetry in the Wave Functions}

Next we shall verify that the structure of the PSM wave function $|I\rangle_{\text {PSM }}$ belonging to different bands obtained by diagonalization is similar to that of the $S U(3)$ wave function $|(\lambda, \mu), I\rangle_{S U(3)}$ belonging to the $S U(3)$ representation $[\lambda, \mu]$ obtained by coupling the $\left[\lambda_{\pi}, 0\right]$ and $\left[\lambda_{\nu}, 0\right]$. Here we consider only the

g-band. The PSM wave functions for the g-band obtained by diagonalizing the Hamiltonian in the basis $\left\{\left(I_{\nu} \otimes I_{\pi}\right) I\right\}$ can be written as

$$
|I\rangle_{\mathrm{PSM}}=\sum_{I_{\nu}, I_{\pi}} f_{\mathrm{PSM}}\left(I_{\nu} I_{\pi} ; I\right)\left|\left(I_{\nu} \otimes I_{\pi}\right) I\right\rangle
$$

and the total $S U(3)$ wave function for the g-band representation $[\lambda, 0]$ can be written as

$$
\begin{aligned}
|(\lambda, \mu), I\rangle_{S U(3)} & =\aleph_{I} \sum_{I_{\nu}, I_{\pi}} a_{I_{\nu}} a_{I_{\pi}}\left\langle I_{\nu} 0 I_{\pi} 0 \mid I 0\right\rangle\left|\left[I_{\nu} \otimes I_{\pi}\right] I\right\rangle \\
& =\sum_{I_{\nu}, I_{\pi}} f_{S U(3)}\left(I_{\nu} I_{\pi} ; I\right)\left|\left(I_{\nu} \otimes I_{\pi}\right) I\right\rangle
\end{aligned}
$$

where $\aleph_{I}$ is the normalization constant

$$
\left|\aleph_{I}\right|^{2}=\frac{1}{\sum_{I_{\nu}, I_{\pi}}\left(a_{I_{\nu}} a_{I_{\pi}}\left\langle I_{\nu} 0 I_{\pi} 0 \mid I 0\right\rangle\right)^{2}} .
$$

The overlap of these two wave functions is

$$
\begin{aligned}
O_{I} & ={ }_{S U(3)}\langle(\lambda, \mu), I \mid I\rangle_{\mathrm{PSM}} \\
& =\sum_{I_{\nu}, I_{\pi}} f_{S U(3)}\left(I_{\nu} I_{\pi} ; I\right) f_{\mathrm{PSM}}\left(I_{\nu} I_{\pi} ; I\right) .
\end{aligned}
$$


Knowing the $S U(3)$ coefficients $f_{S U(3)}$ from $a_{I_{\nu}}$ and $a_{I_{\pi}}$, and the PSM coefficients $f_{\mathrm{PSM}}$ from numerical diagonalization, we are able to compute their overlap. The calculated overlaps $O_{I}$ are listed in Table $2 \square$. All the numbers are very close to 1 , indicating a strong overlap of the PSM wave functions with the $S U(3)$ wave functions.

\subsection{SU(3)-like Systematics of $B(E 2, I \rightarrow I-2)$ values}

The $B(E 2)$ values are useful to determine the collectivity of a band. In the discussion of the last section, the numerically calculated PSM states were classified using group theoretical methods. We shall now verify this classification with $B(E 2)$ calculations using PSM wave function, which will allow us to classify PSM states into collective bands. Since we have proved the similarity of the PSM wave functions to the $S U(3)$ ones, we expect to obtain similar $B(E 2)$ values if we make a corresponding calculation using $S U(3)$ wave functions.

The $B(E 2)$ transitions are calculated as

$$
B\left(E 2, I_{i} \rightarrow I_{f}\right)=\frac{\left|\left\langle I_{f}\left\|\mathcal{T}^{2}\right\| I_{i}\right\rangle\right|^{2}}{2 I_{i}+1}
$$

where the operator $\mathcal{T}^{2}=\mathcal{T}_{\nu}^{2}+\mathcal{T}_{\pi}^{2}$ is related to the quadrupole operators by

$$
\begin{aligned}
& \mathcal{T}_{\nu}^{2}=e_{\nu}^{\text {eff }} \sqrt{\frac{5}{16 \pi}} Q_{\nu}^{2}, \\
& \mathcal{T}_{\pi}^{2}=e_{\pi}^{\text {eff }} \sqrt{\frac{5}{16 \pi}} Q_{\pi}^{2} .
\end{aligned}
$$

In the calculations, we have used the usual effective charges $e_{\nu}^{\text {eff }}=0.5 e$ and $e_{\pi}^{\text {eff }}=1.5 e$. By employing the PSM wave functions of Eq. (17), the reduced matrix element appearing in Eq. (21) can be evaluated as

$$
\begin{aligned}
\left\langle I_{f}\left\|\mathcal{T}^{2}\right\| I_{i}\right\rangle= & \sum_{I_{f}\left(I_{f, \nu}, I_{f, \pi}\right)} \sum_{I_{i}\left(I_{i, \nu}, I_{i, \pi}\right)} f_{f}\left(I_{f, \nu} I_{f, \pi} ; I_{f}\right) f_{i}\left(I_{i, \nu} I_{i, \pi} ; I_{i}\right) \\
& \left\langle\left(I_{f, \nu} \otimes I_{f, \pi}\right) I_{f}\left\|\mathcal{T}_{\nu}^{2}+\mathcal{T}_{\pi}^{2}\right\|\left(I_{i, \nu} \otimes I_{i, \pi}\right) I_{i}\right\rangle,
\end{aligned}
$$

1 A similar calculation for the overlaps for the PSM wave functions $|I\rangle_{P S M}$ belonging to higher bands with the $S U(3)$ wave functions of the representations [44,2], $[40,4]$, etc. requires calculation of $S U(3)$ Clebsch-Gordon coefficients. The proof should be straightforward and is not given here. 
in which the matrix elements for a coupled system can be explicitly expressed as $[10]$

$$
\begin{aligned}
\left\langle\left[I_{f, \nu} \otimes I_{f, \pi}\right] I_{f}\right. & \left.\left\|\mathcal{T}_{\nu}^{2}\right\|\left[I_{i, \nu} \otimes I_{i, \pi}\right] I_{i}\right\rangle=\sqrt{\left(2 I_{i}+1\right)\left(2 I_{f}+1\right)} \\
& (-)^{I_{f, \nu}+I_{f, \pi}+I_{i}} \mathcal{W}\left(I_{i, \nu} I_{i, \pi} 2 I_{f} ; I_{i} I_{f, \nu}\right)\left\langle I_{f, \nu}\left\|\mathcal{T}_{\nu}^{2}\right\| I_{i, \nu}\right\rangle \delta_{I_{f, \pi} I_{i, \pi}}, \\
\left\langle\left[I_{f, \nu} \otimes I_{f, \pi}\right] I_{f}\right. & \left.\left\|\mathcal{T}_{\pi}^{2}\right\|\left[I_{i, \nu} \otimes I_{i, \pi}\right] I_{i}\right\rangle=\sqrt{\left(2 I_{i}+1\right)\left(2 I_{f}+1\right)} \\
& (-)^{I_{i, \nu}+I_{i, \pi}+I_{f}} \mathcal{W}\left(I_{i, \nu} I_{i, \pi} I_{f} 2 ; I_{i} I_{f, \pi}\right)\left\langle I_{f, \pi}\left\|\mathcal{T}_{\pi}^{2}\right\| I_{i, \pi}\right\rangle \delta_{I_{f, \nu} I_{i}(24)}
\end{aligned}
$$

In the $S U(3)$ model, assuming the transition operator $\mathcal{T}^{2}=\alpha P^{2}$, where $P^{2}$ is the generator of the coupled $S U(3)^{\nu+\pi}$, the $B(E 2)$ formula for the intra-band transition is

$$
B\left(E 2, I_{i} \rightarrow I_{f}\right)=\frac{2 I_{f}+1}{2 I_{i}+1} \alpha^{2}\left|\left\langle(\lambda \mu) K I_{i},(11) 2 \|(\lambda \mu) K I_{f}\right\rangle\right|^{2} C(\lambda, \mu),(25
$$

where $\left\langle(\lambda \mu) K I_{i},(11) 2 \|(\lambda \mu) K I_{f}\right\rangle$ is the $S U(3) \supset R(3)$ Wigner coefficient, and $\alpha$ is a parameter of the effective transition operator in the $S U(3)$ model determined by fitting data. The inter-band transition rate is zero. In the symmetry limit, if the transition operator is not a generator of $S U(3)^{\nu+\pi}$ or there exists small symmetry breaking in the wavefunction, the inter-band transition rate would be expected to be small but not zero.

Generally, the shell-model E2 operator has different effective charges for neutrons and protons and as such is not a generator of the coupled $S U(3)^{\nu+\pi}$. We note, however, that Eq. (25) is still a good approximation for this more general $E 2$ operator through the inclusion of a factor $\left(e_{\nu} \lambda_{\nu}+e_{\pi} \lambda_{\pi}\right) /\left(\lambda_{\nu}+\lambda_{\pi}\right)$ in the proportionality constant $\alpha$. Different neutron and proton effective charges (or gyromagnetic ratios for $M 1$ 's) will also give rise to inter-band transitions, which is an essential mechanism to obtain the strong $M 1$ 's from scissors states to ground band.

The $B(E 2)$ values calculated by the PSM are shown in Fig. 3, in comparison with the $S U(3)$ results and the experimental data [27]. For the g-band (labeled as the first $0^{+}$band in the tables), good agreement is found for all states except for the transition $6^{+} \rightarrow 4^{+}$. Besides the g-band, we have calculated the $B(E 2)$ 's for the first $1^{+}$band, the second $0^{+}$band, and the first $2^{+}$band (see Fig. 1). The values displayed in Table 3 confirm strong collectivity in each of these intra-transition bands and is in good agreement with what found in the $S U(3)$ model. Linking $B(E 2)$ values between any of two bands are also calculated, with the results displayed in Table 4 . These inter-band $B(E 2)$ values are typically two orders of magnitude smaller than the values for the intra-band transitions. From these calculations, the sets of nearly degenerate 
bands seen in the PSM calculations of Fig. 1 (for example, the second $0^{+}$ band and the first $2^{+}$band) can be separated easily. All these results strongly support the conclusion that the structure of well deformed nuclei resulting from PSM calculations indeed possesses a very strong $S U(3)$ symmetry.

\section{Comments on the Emergent SU(3) Symmetry}

The connection between $S U(3)$ symmetry and nuclear rotation has a long history. It was first explored by Elliott in his classic 1958 papers [1] for $s d$-shell nuclei. This idea was later extended to heavy systems using the pseudo- $S U(3)$ model [31], which showed that it is possible to represent approximately the ground band of a deformed nucleus by one leading $S U(3)$ representation belonging only to the normal parity nucleons in a single major shell. However this model has difficulty in reproducing $\beta$-bands of heavy nuclei, and quantitative results generally do not appear in simple symmetry limits and therefore require numerical calculations. Later, the interacting boson model (IBM) [32] demonstrated that nuclear rotational motion including $\beta$ - and $\gamma$-bands can be described by an $S U(3)$ dynamical symmetry based on $s-d$ or $s-d-g$ bosons. The fermion dynamical symmetry model (FDSM), based on the $S$ - $D$ fermion pairs of normal parity nucleons in a major shell, demonstrated analytically the equivalence between the particle-rotor model and the $S U(3)$ dynamical symmetry limit of the FDSM when particle number $n \rightarrow \infty$; if the Pauli effect is neglected (by assuming the shell pair-degeneracy $\Omega \rightarrow \infty$ ) the FDSM reduces to the IBM (see Section 3 and 4 in Ref. [28]).

However, all the above-mentioned models are algebraic and the possibility for symmetries arises naturally in them. The extended PSM described here has not built an explicit $S U(3)$ symmetry into the problem, and no free parameters have been adjusted. Nevertheless, the spectra and wavefunctions obtained from the PSM calculations can again be well classified using the representation theory of the $S U(3)$ group. The $S U(3)$ symmetry just emerges naturally at the macroscopic level from a shell model diagonalization in the basis obtained by angular momentum projection from a deformed intrinsic state. This is a nontrivial result because our study is not confined in the g-band, but extended up to high spins and high excitations, and because the correspondence has been demonstrated not only at the spectral level, but in the wavefunctions and electromagnetic transition rates too. This strongly indicates that a well deformed nucleus is in fact a very good $S U(3)$ rotor.

Now we may ask an important question: what is the nature of the $S U(3)$ symmetry that emerges from the PSM? There are many $S U(3)$ models. Mathematically, they are equivalent if their representations are the equivalent. Phys-

ically, the models differ in the microscopic basis on which the symmetry is 
built, and have different permissible $S U(3)$ irreps, and thus different band structures. The emergent $S U(3)$ symmetry we have found here is in principle consistent with any $S U(3)$ symmetry model that has allowed representations consistent with the observed states and band structure. We emphasize that this is a physical rather than mathematical criterion, for it is the physical input to an $S U(3)$ model that determines its allowed irreps. Therefore, to answer the question of which $S U(3)$ model is consistent with the symmetry of the well deformed rotor that we have explored in this paper, one should look at the entire band structure and not just the ground-state rotational band.

As we have already demonstrated, the $S U(3)$ symmetry that emerges from the PSM for ${ }^{168} \mathrm{Er}$ corresponds to effective $\left\{\left[\lambda_{\nu}=32,0\right] \otimes\left[\lambda_{\pi}=16,0\right]\right\}(\lambda, \mu) I$ irreps for neutrons and protons, respectively. Among the $S U(3)$ models being employed in nuclear structure physics, the FDSM (or equivalently the IBM that results if Pauli effects are neglected in the FDSM) can naturally accommodate such a representation if the shell degeneracy $\Omega$ is large enough [28]. As can be easily checked, no other currently existing $S U(3)$ fermion model permits naturally such an $S U(3)$ representation. For example, the leading $S U(3)$ irreps for ${ }^{168} \mathrm{Er}$ given by the pseudo- $S U(3)$ model [31] would be $\left\{\left[\lambda_{\nu}^{\text {pseudo }}=20, \mu_{\nu}^{\text {pseudo }}=4\right] \otimes\left[\lambda_{\pi}^{\text {pseudo }}=10, \mu_{\pi}^{\text {pseudo }}=4\right]\right\}(\lambda, \mu) I$, which will give a band structure very different from what we have seen in Fig. 1. Thus, we conclude that in a model such as the pseudo- $S U(3)$, the $S U(3)$ spectrum found in the numerical PSM calculation cannot be reproduced by any simple symmetry limit and could emerge (if at all) only from a large mixing of irreps through numerical diagonalization.

However, it should be noted that although we have demonstrated that the $S U(3)$ symmetry that emerges from the PSM is of FDSM-type (or the IBMtype if Pauli effects are omitted), this does not necessarily mean that the simplest single-major shell FDSM can accommodate such an $S U(3)$ symmetry microscopically. If we assume that the $S U(3)$ symmetry which emerges for ${ }^{168} \mathrm{Er}$ in the present PSM calculation is the fermion dynamical $S U(3)$ symmetry of Ref. [28] based on $S$ - $D$ fermion pairs, the $S U(3)$ quantum numbers $\lambda_{\nu}$ and $\lambda_{\pi}$ have a very simple interpretation. They are the effective number of neutrons and protons in the valence space, $\lambda_{\nu}=n_{\nu}^{\text {eff }}$ and $\lambda_{\pi}=n_{\pi}^{\text {eff }}$, which form the coherent $S-D$ pairs and are responsible for the collective rotation. In the simplest implementations of the FDSM, the model space is restricted to one major shell for protons and neutrons. The effective neutron (proton) number $n_{\nu}^{\text {eff }}\left(n_{\pi}^{\text {eff }}\right)$ is just the number of neutrons (protons) in the normal-parity levels of a single major shell, which is obviously too small to satisfy the requirement of $n_{\nu}^{\text {eff }} \geq 32$ and $n_{\pi}^{\text {eff }} \geq 16$ for the ${ }^{168} \mathrm{Er}$ case. In order to accommodate the $\left\{\left[\lambda_{\nu}=32,0\right] \otimes\left[\lambda_{\pi}=16,0\right]\right\}(\lambda, \mu) I S U(3)$ symmetry, the one-major shell FDSM has to be extended to a multi-major shell FDSM. Namely, the coherent $S-D$ pairs should be redefined in a multi-major shell space. This has already been discussed extensively in conjunction with the extension of the FDSM to 
the description of superdeformation, and much of that discussion applies to the present case.

One should note in this regard that there is a conceptual distinction between the $S U(3)$ symmetry that emerged from the PSM diagonalized in multiple shells and that which arises in the one-major shell FDSM. In the PSM calculations the $S U(3)$ symmetry arises from the explicit dynamical participation of both normal and abnormal-parity nucleons, while in the symmetry limit of the single one-major shell FDSM the $S U(3)$ symmetry itself arises entirely from the normal-parity nucleons and the abnormal parity orbital enters only implicitly through the Pauli effect and by renormalizing the $S U(3)$ parameters. Thus, the multi-major shell FDSM is also necessary to resolve this conceptual ambiguity. The reason that the one-major shell FDSM treats the contribution of the abnormal-parity component to the collective motion differently from normal parity orbitals is because in a single major shell there is only one abnormal parity level. It has been shown that a single- $j$ shell does not have enough quadrupole collectivity (the possibility to form a $D$ pair in the $i_{\frac{13}{2}}$ intruder level compared to that in the corresponding normal-parity levels is only $\frac{6}{46}$; see Ref. [33]). When the FDSM is extended to a three-shell valence space as in the PSM, the situation will be changed. A bunch of abnormalparity levels, which are located just below the normal-parity levels, will open up. Abnormal-parity nucleons thus have enough collectivity to form coherent $D$ pairs and participate in collective motion. This idea has already been developed in the extension of the FDSM to superdeformation.

Although the basic theme of the present work is that the $S U(3)$ symmetry-like features emerge for deformed nuclei from a realistic Hamiltonian which has no such symmetry at a microscopic level, it is tempting to look for the microscopic basis underlying the emergent $S U(3)$ symmetry. The preceding arguments give us strong reason to speculate that this emergent $S U(3)$ symmetry is just a manifestation of the FDSM operating over a three-shell valence space.

\section{Comments on the Scissors Mode Vibrations}

A second important consequence of our study is that the rotational states that we have just described in terms of an $S U(3)$ symmetry can be regrouped and interpreted as phonon vibrations (even though no explicit vibrational information has been given to the calculation). A standard signature of ideal harmonic vibrational motion is the appearance of an equally spaced spectrum of energy levels with a characteristic degeneracy pattern. A similar connection between $S U(3)$ symmetry and vibration in rotating nuclei was pointed out by $\mathrm{Wu}$ et al. for $\beta$ - and $\gamma$-vibrations [34]. In the present case, we have clearly shown that the band heads of the different $S U(3)$ representations $[\lambda, \mu]$ ob- 
tained by coupling the proton-neutron representations $\left[\lambda_{\pi}, 0\right]$ and $\left[\lambda_{\mu}, 0\right]$ tend to be equally spaced. We have also shown that the spectra of the states obtained by PSM diagonalization is dominated by nearly equally spaced bands (apart from a small anharmonicity). Therefore it is appropriate to call these "vibrational bands". Physically the only vibrational mode allowed for protons and neutrons that retain their individual $S U(3)$ irreps $\left[\lambda_{\tau}, 0\right]$ (this algebraic restriction is related to a geometrical requirement of fixed deformation for both neutrons and protons) is the "scissors mode". The scissors mode corresponds geometrically to an oscillation in the relative orientation angle of the quadrupole-deformed neutron and proton potentials.

Traditionally, collective vibrations appear as a consequence of small oscillations around the equilibrium in the deformed potential, as we know from the physics of $\beta$ - and $\gamma$-vibrations [10]. Theories of the RPA type allow superposition of states displaced around the equilibrium, thus describing the physics of small oscillations, as required for $\beta$ - and $\gamma$-vibrations. However, the collective vibrations considered here originate from relative motion between proton and neutron systems at fixed deformation. As long as one does not decompose the neutron and proton degrees of freedom, the physics discussed in our work is not contained in such theories.

On the other hand, it is clear that the present results do not describe $\beta$ and $\gamma$-spectra because the $\beta$ - and $\gamma$-vibrational modes do not belong to the present configuration space. This can be seen clearly with the aid of the preceding $S U(3)$ classification of the PSM states. The $\beta$ - and $\gamma$-vibrations (the quadrupole phonon or spin- $2 \hbar$ excitations), correspond to a coherent superposition of the $\left[\left(\lambda_{\nu}-2 \mu, \mu\right) \otimes\left(\lambda_{\pi}, 0\right)\right]$ and $\left[\left(\lambda_{\nu}, 0\right) \otimes\left(\lambda_{\pi}-2 \mu, \mu\right)\right]$ representations with $\mu$ equal to a non-zero even integer. In other words, either the neutron or the proton core (or both), must be excited. It is easy to show from Eq. (11) that the dominant $S U(3)$ representation for the lowest $\beta$ - and $\gamma$-bands is $\left[\left(\lambda_{\nu}-4,2\right) \otimes\left(\lambda_{\pi}, 0\right)\right](\lambda-4,2)$ if $\lambda_{\nu}>\lambda_{\pi}$. The $\beta$-band head energy is given by

$$
E_{\beta 0}-E_{\text {g.s. }}=-3 \lambda_{\nu} \chi_{\nu}^{\mathrm{eff}}+2 \hbar \omega_{\infty}, \quad \hbar \omega_{\infty}=\frac{3}{2} \lambda_{m} \chi_{\nu \pi}^{\mathrm{eff}}
$$

Thus the $\beta$-vibrational band head energy is significantly lower than the energy $E_{2 \text { nd }-0^{+}}=2 \hbar \omega_{\infty}$ of the lowest $K=0^{+}$band of the scissors mode excitation shown in Fig. 1 which corresponds to the $\left[\left(\lambda_{\nu}, 0\right) \otimes\left(\lambda_{\pi}, 0\right)\right]\left(\lambda_{m}-4,2\right)$ representation. In fact, $E_{\beta 0}$ will be lower than even the energy $E_{1 \mathrm{st}-1^{+}}$of the band head of the first $1^{+}$scissors mode $E_{1 s t-1^{+}}=\hbar \omega_{\infty}+2 A$, if the condition

$\chi_{\nu}^{\mathrm{eff}}>\frac{\lambda_{m}}{2 \lambda_{\nu}} \chi_{\nu \pi}^{\mathrm{eff}}$ is fulfilled. In the case of ${ }^{168} \mathrm{Er}$ discussed here, this condition becomes $\chi_{\nu}^{\mathrm{eff}}>0.75 \chi_{\nu \pi}^{\mathrm{eff}}$.

This means that, unlike the scissors mode, the $\beta$ - and $\gamma$-vibrations do not correspond to relative motion between fixed neutron and proton fields, but 
to an internal collective excitation of neutrons or/and protons. This picture is consistent with the conventional shape vibration picture [10]. Therefore, to obtain the classical $\beta$ - and $\gamma$-bands within the present framework one must build a richer set of correlations into the vacuum. In principle, this could be achieved by mixing a large set of multi-quasiparticle states, but in practice, the configuration space may be too large to handle if there is substantial collectivity in these modes. A more efficient way to introduce these states is to build those degrees of freedom into the intrinsic basis. Very recently, $\gamma$-band and multi-phonon $\gamma$-vibrational states in rare-earth nuclei have been obtained by Sun et al. [12] by using three-dimensional angular momentum projection on triaxially deformed potential [35].

\section{The First $1^{+}, 2^{+}$, and the Second $0^{+}$Bands}

Several lowest excited bands appearing in Fig. 1 warrant further discussion. In Fig. 4 we plot these bands, together with the g-band from the PSM calculations and from experiment [27]. There are other observed low-lying collective states in this nucleus (for example, the $2^{+} \gamma$-band starting at $0.821 \mathrm{MeV}$ [27], which was extensively studied in the PSM framework in Ref. [12]). Discussion of these states is beyond the scope of the present paper, and therefore, we omit plotting them in Fig. 4.

The first $1^{+}$band corresponds to a 1-phonon excitation with the excitation energy relative to the ground state depending on the interaction strengths used in the calculation. The good agreement of the calculated g-band with data (and similar results for many other calculations in this mass region [5]) suggests that the strengths we use here are realistic. These excitations are due to an $S U(3)$ coupling $\left(n_{\nu}, 0\right) \otimes\left(n_{\pi}, 0\right)$ in which both neutron and proton intrinsic systems remain in the ground states; thus they are related physically to relative motion between neutrons and protons. We conclude that this may be the $1^{+}$scissors mode band previously suggested in other models [13-17]. Our obtained bandhead of this $1^{+}$band is at an excitation of about $3 \mathrm{MeV}$, reasonably within the energy range of experimental observations for scissors mode [36].

The present results indicate that the PSM provides a microscopic framework in which collective modes that may be closely identified with those proposed in earlier geometrical and algebraic descriptions emerge as the lowest excitations. Furthermore, it is already well established that the PSM describes structures built on quasiparticle excitation very well [5]. Therefore, the extension of the present calculations to a larger basis including 2 and possibly 4 quasiparticle excitations of the $\mathrm{n}-\mathrm{p}$ coupled vacuum will provide a microscopic formalism in which collective and quasiparticle degrees of freedom enter on an equal 
footing. Such calculations are possible and are presently being explored. We may expect that the long-debated question of whether the observed $1^{+}$states are collective or two-quasiparticle in nature may then be resolved through such quantitative calculations of this sort.

The other excited bands are generalizations of the scissors mode corresponding to multi-phonon excitations. In Fig. 4, the next two bands at $6.5 \mathrm{MeV}$ excitation energy are nearly degenerate 2-phonon states, corresponding to the coupling of two $1 \hbar$-phonons to total spins $0 \hbar$ and $2 \hbar$. These are theoretically predicted multi-phonon excitations of the scissors mode, and have not to our knowledge been seen experimentally. Although the level density is expected to be high at that excitation energy and symmetry breaking will fractionate the

strength, these predicted states might be detectable in the new generation of modern detectors.

\section{Summary}

We have found many new collective states in a shell model diagonalization based on separately projected neutron and proton Nilsson + BCS vacuum states. We have shown that these states exhibit an almost perfect $S U(3)$ symmetry, both in their spectra and their wavefunctions. We have shown also that these states can be classified systematically in a phonon spectrum with weak anharmonicity. Among these states, the lowest $1^{+}$band at about $3 \mathrm{MeV}$ corresponds to the scissors mode predicted in a classical geometrical picture. The PSM is a shell model diagonalization without explicit $S U(3)$ symmetries. However, the quantitative agreement with an $S U(3)$ model provides an algebraic fermion classification scheme for the states obtained from the PSM diagonalization, and suggests that the projected BCS vacuum for a well-deformed system is very close to the $S U(3)$ dynamical symmetry limit of an $S$ - $D$ pair fermion system. This in turn implies a good boson algebraic symmetry if Pauli effects may be ignored. Finally, we have proposed that the extension of the present calculations to include quasiparticle excitations provides a quantitative framework to determine whether "scissors mode" $1^{+}$states are more properly viewed as collective excitations or as quasiparticle states.

The present paper deals only with even-even nuclear system. However, our preliminary results [37] have shown that similar conclusions can also be drawn for odd- and odd-odd nuclei.

Y.S. thanks Professor Gui-Lu Long of Department of Physics of Tsinghua University for warm hospitality, where the final version of this paper was completed. K.H.B. thanks Dr. S. Raman of Oak Ridge National Laboratory for partial support. 


\section{References}

[1] J.P. Elliott, Proc. Roy. Soc. (Lond.) A245 (1958) 128; A245 (1958) 562.

[2] K. H. Bhatt, J. C. Parikh and J. B. McGrory, Nucl. Phys. A224 (1974) 301.

[3] D.M. Brink, B. Buck, R. Huby, M.A. Nagarajan and N. Rowley, J. Phys. G13 (1987) 629.

[4] Y. Sun, C.-L. Wu, K. Bhatt, M. Guidry and D.H. Feng, Phys. Rev. Lett. 80 (1998) 672.

[5] K. Hara and Y. Sun, Int. J. Mod. Phys. E4 (1995) 637.

[6] E. Caurier, A.P. Zuker, A. Poves and G. Martínez-Pinedo, Phys. Rev. C50 (1994) 225.

[7] Y. Sun, J.-y. Zhang and M. Guidry, Phys. Rev. Lett. 78 (1997) 2321.

[8] Y. Sun, J.-y. Zhang, M. Guidry and C.-L. Wu, Phys. Rev. Lett. 83 (1999) 686.

[9] G.-L. Long and Y. Sun, Phys. Rev. C63 (2001) 021305(R).

[10] A. Bohr and B.R. Mottelson, Nuclear Structure (W.A.Benjamin Inc., New York, 1975).

[11] J.L. Egido, H.J. Mang and P. Ring, Nucl. Phys. A339 (1980) 390.

[12] Y. Sun, K. Hara, J.A. Sheikh, J.G. Hirsch, V. Velázquez and M. Guidry, Phys. Rev. C61 (2000) 064323.

[13] T. Suzuki and D.J. Rowe, Nucl. Phys. A289 (1977) 461.

[14] R.R. Hilton, Z. Phys. A316 (1984) 121, and references cited therein.

[15] N. Lo Iudice and F. Palumbo, Phys. Rev. Lett. 41 (1978) 1532.

[16] R. Nojarov, Z. Bochnacki and A. Faessler, Z. Phys. A324 (1986) 289.

[17] F. Iachello, Phys. Rev. Lett. 53 (1984) 1427.

[18] J. Dobaczewski, W. Nazarewicz, J. Skalski and T. Werner, Phys. Rev. Lett. 60 (1988) 2254, and references cited therein.

[19] L.S. Kisslinger and R.A. Sorensen, Rev. Mod. Phys. 35 (1963) 853; M. Baranger and K. Kumar, Nucl. Phys. A110 (1968) 490; D.R. Bes and R.A. Sorensen, Adv. Nucl. Phys. 2, (1969) 129.

[20] M. Dufour and A.P. Zuker, Phys. Rev. C54 (1996) 1641.

[21] S.G. Nilsson et al., Nucl. Phys. A131 (1969) 1.

[22] P. Ring and P. Schuck, The Nuclear Many Body Problem (Springer-Verlag, New York, 1980). 
[23] A.R. Edmonds, Angular Momentum in Quantum Mechanics, 3rd Edition (Princeton University Press, Princeton, 1974).

[24] R. Bengtsson, S. Frauendorf and F.-R. May, Atom. Data and Nucl. Data Tables 35 (1986) 15.

[25] K. Hara and S. Iwasaki, Nucl. Phys. A348 (1980) 200.

[26] K. Hara and Y. Sun, Nucl. Phys. 529 (1991) 445.

[27] B. Kotliński, D. Cline, A. Bäcklin, K.G. Helmer, A.E. Kavka, W.J. Kernan, E.G. Vogt, C.Y. Wu, R.M. Diamond, A.O. Macchiavelli and M.A. Deleplanque, Nucl. Phys. A517 (1990) 365.

[28] C.-L. Wu, D.H. Feng and M. W. Guidry, Adv. in Nucl. Phys. 21 (1994) 227.

[29] J.D. Vergados, Nucl. Phys. A111 (1968) 681.

[30] S. Kahane, S. Raman and K.H. Bhatt, Phys. Rev. 55 (1997) 2885.

[31] A. Arima, M. Harvey and K. Shimizu, Phys. Lett. B30 (1969) 517; K.T. Hecht and A. Adler, Nucl. Phys. A137 (1969) 129; R.D. Ratna Raju, J.P. Draayer and K.T. Hecht, Nucl. Phys. A202 (1973) 433.

[32] A. Arima and F. Iachello, Ann. Phys. 99 (1976) 253; 111 (1978) 201; 115 (1978) 325.

[33] M. W. Guidry and C.-L. Wu, J. Mod. Phys E2 (1993) 17.

[34] C.-L. Wu, M.W. Guidry, D.H. Feng, and J.-Q. Chen, Fizika 22 (1990) 123.

[35] J.A. Sheikh and K. Hara, Phys. Rev. Lett. 82 (1999) 3968.

[36] C. Schlegel, P. von Neumann-Cosel, A. Richter and P. Van Isacker, Phys. Lett. B375 (1996) 21.

[37] Y. Sun et al., to be published. 
Table 1

The calculated angular momentum distribution in the intrinsic vacuum states of Nilsson-BCS, $\left|C_{I}\right|^{2}$, for the first nine angular momenta, for the neutron vacuum $\left|0_{\nu}\right\rangle$, the proton vacuum $\left|0_{\pi}\right\rangle$, and the product vacuum $|0\rangle=\left|0_{\nu}\right\rangle\left|0_{\pi}\right\rangle$.

\begin{tabular}{|cccc|} 
Spin I & $\left|C_{I_{\nu}}\right|^{2}$ & $\left|C_{I_{\pi}}\right|^{2}$ & $\left|C_{I}\right|^{2}$ \\
\hline 0 & 0.0313 & 0.0591 & 0.0203 \\
2 & 0.1419 & 0.2452 & 0.0954 \\
4 & 0.2041 & 0.2877 & 0.1486 \\
6 & 0.2078 & 0.2158 & 0.1712 \\
8 & 0.1695 & 0.1186 & 0.1647 \\
10 & 0.1161 & 0.0504 & 0.1381 \\
12 & 0.0684 & 0.0171 & 0.1032 \\
14 & 0.0352 & 0.0047 & 0.0696 \\
16 & 0.0159 & 0.0011 & 0.0426
\end{tabular} \mid

Table 2

The overlap of the PSM and the $S U(3)$ wave functions.

\begin{tabular}{|cc|} 
Spin $I$ & Overlap $O_{I}$ \\
\hline 0 & 0.9686 \\
2 & 0.9683 \\
4 & 0.9677 \\
6 & 0.9671 \\
8 & 0.9668 \\
10 & 0.9669 \\
12 & 0.9673 \\
14 & 0.9678 \\
16 & 0.9680 \\
18 & 0.9676
\end{tabular}


Table 3

Calculated $\mathrm{B}(\mathrm{E} 2, I \rightarrow I-2)$ values (in $\left.e^{2} b^{2}\right)$ for several excited bands with PSM wave functions.

\begin{tabular}{|ccccc|} 
Spin I & 1 st $0^{+}$ & 1st $1^{+}$ & 2nd $0^{+}$ & 1 st $2^{+}$ \\
\hline 2 & 1.104 & & 1.009 & \\
4 & 1.578 & 1.249 & 1.383 & 0.599 \\
6 & 1.739 & 1.541 & 1.469 & 1.196 \\
8 & 1.821 & 1.667 & 1.550 & 1.487 \\
10 & 1.872 & 1.735 & 1.617 & 1.639 \\
12 & 1.906 & 1.778 & 1.661 & 1.718 \\
\hline 3 & & 0.917 & & \\
5 & & 1.459 & & 0.969 \\
7 & & 1.647 & & 1.339 \\
9 & & 1.741 & & 1.512 \\
11 & & 1.797 & & 1.609
\end{tabular}

Table 4

Calculated $\mathrm{B}(\mathrm{E} 2, I \rightarrow I-2)$ values (in $\left.e^{2} b^{2}\right)$ for linking transitions between bands.

\begin{tabular}{|ccccc|} 
Spin I & 1 st $1^{+} \rightarrow 1$ st $0^{+}$ & 2 nd $0^{+} \rightarrow 1$ st $1^{+}$ & 1 st $2^{+} \rightarrow 2$ nd $0^{+}$ & 1 st $2^{+} \rightarrow 1$ st $1^{+}$ \\
\hline 2 & 0.017 & & 0.007 & \\
4 & 0.020 & 0.016 & 0.063 & 0.017 \\
6 & 0.020 & 0.026 & 0.094 & 0.009 \\
8 & 0.020 & 0.032 & 0.055 & 0.004 \\
10 & 0.020 & 0.034 & 0.022 & 0.002 \\
12 & 0.019 & 0.035 & 0.008 & 0.001 \\
\hline 3 & & & & 0.021 \\
5 & & & & 0.024 \\
7 & & & 0.023 \\
9 & & & 0.022 \\
11 & & & & 0.021
\end{tabular}


Fig. 1. Spectrum of collective excitations corresponding to coupled rotation of neutrons and protons. Symbols are calculated by the Projected Shell Model; dashed lines are calculated by an $S U(3)$ Symmetry Model. For those symbols, "•" represents states having $K=0$ and 1, "o" having $K=2$ and 3, "-" having $K=4$ and 5, and " $\times$ " having $K=6$ and 7. Many states are covered by other states because of the high level of degeneracy. The degeneracy is indicated explicitly for the $(40,4)$ states.

Fig. 2. Comparison of angular momentum distribution in the intrinsic vacuum states of Nilsson-BCS calculated in the PSM and the $S U(3)$ representation. The curves connect the PSM values and the symbols represent the $S U(3)$ values.

Fig. 3. Comparison of the calculated $\mathrm{B}(\mathrm{E} 2, I \rightarrow I-2)$ values from the PSM with experimental data for ${ }^{168} \mathrm{Er}$ g-band[27] and the $S U(3)$ limit (calculated from Eq. (25) with the parameter $\alpha=0.0683$ ).

Fig. 4. Spectrum of the ground band, the first $1^{+}$and $2^{+}$, and the second $0^{+}$ collective bands in ${ }^{168} \mathrm{Er}$. 


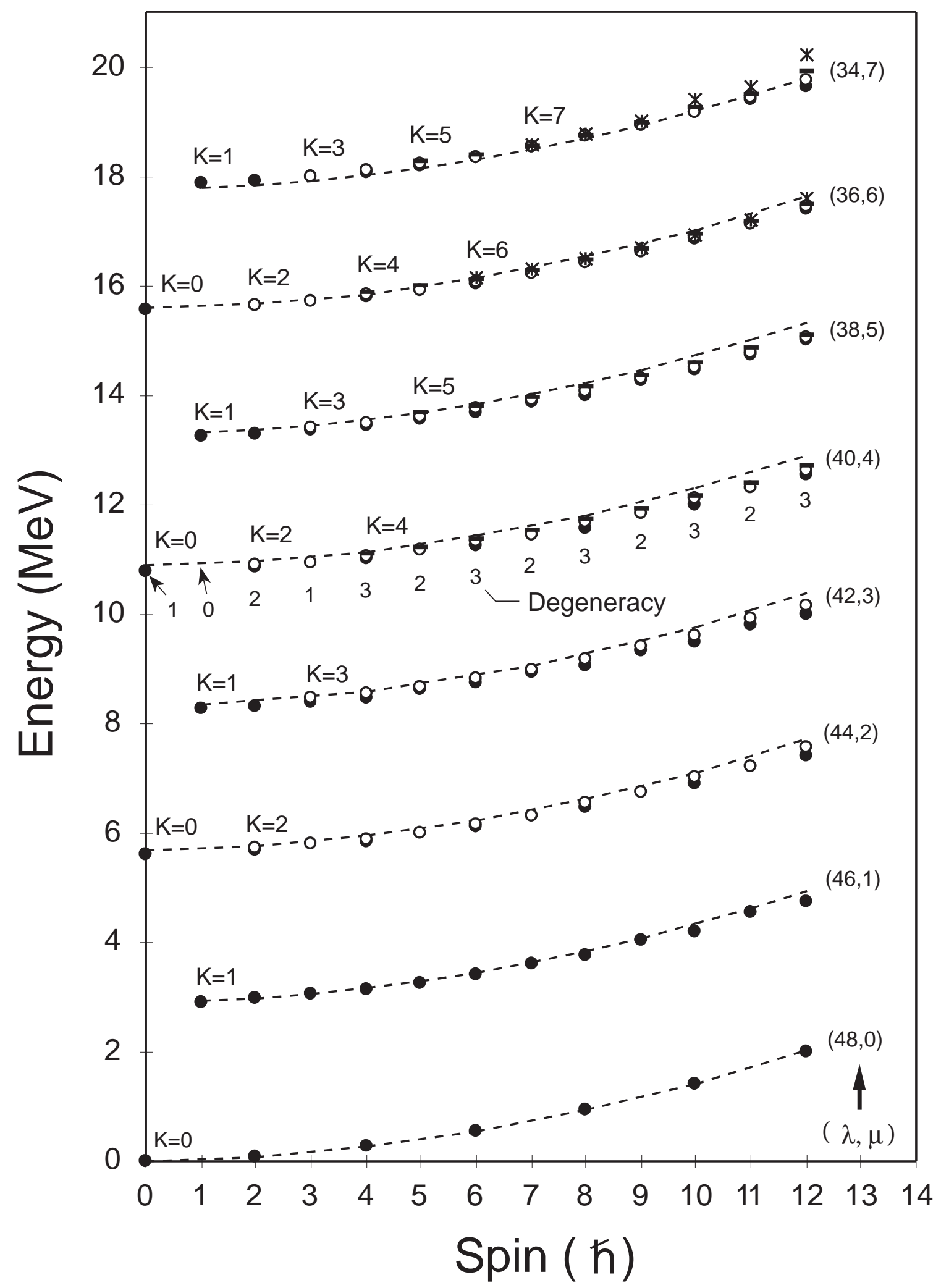

Fig. 1, Sun, Wu, Bhatt and Guidry 


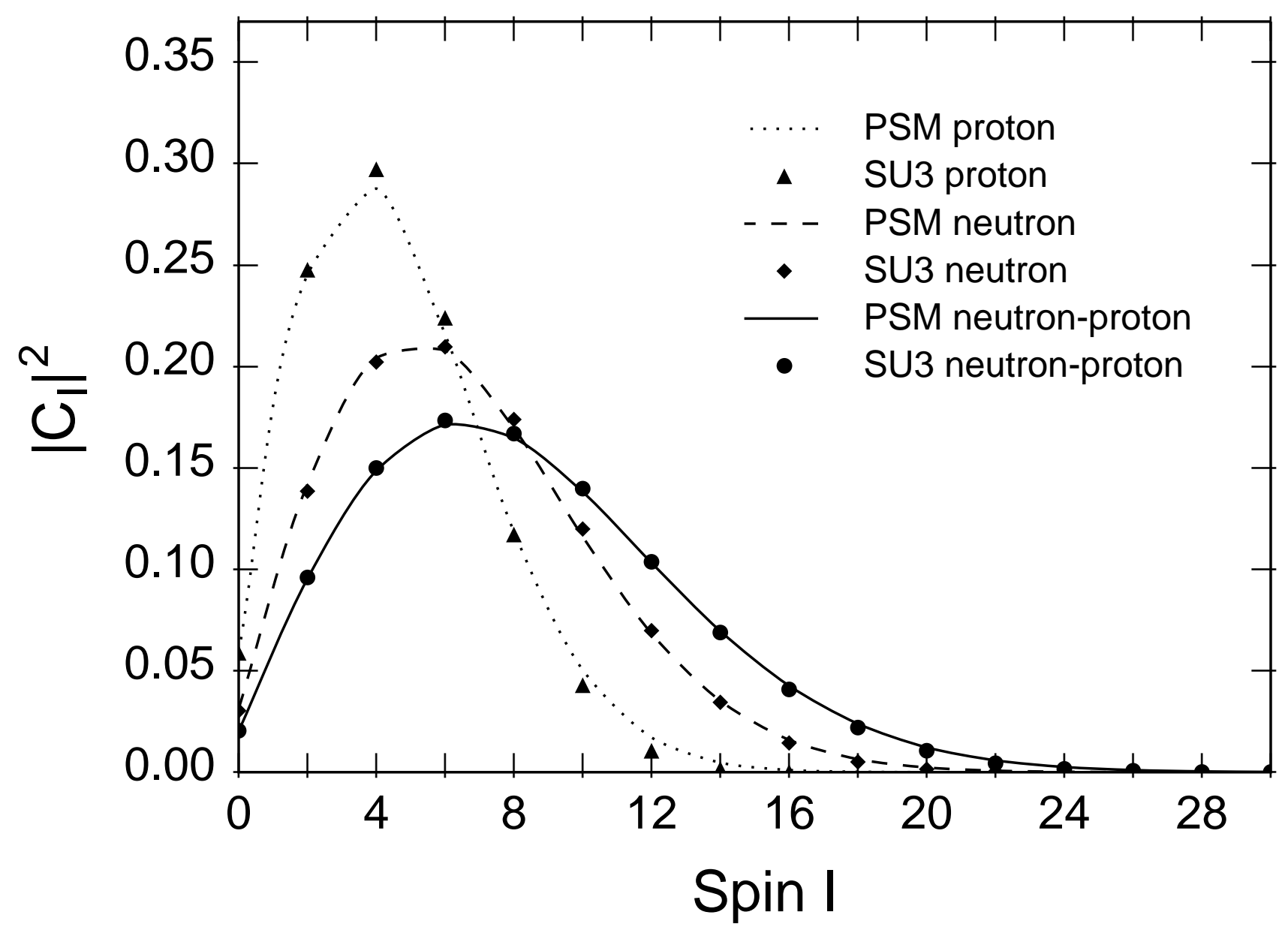

Fig. 2, Sun, Wu, Bhatt and Guidry 

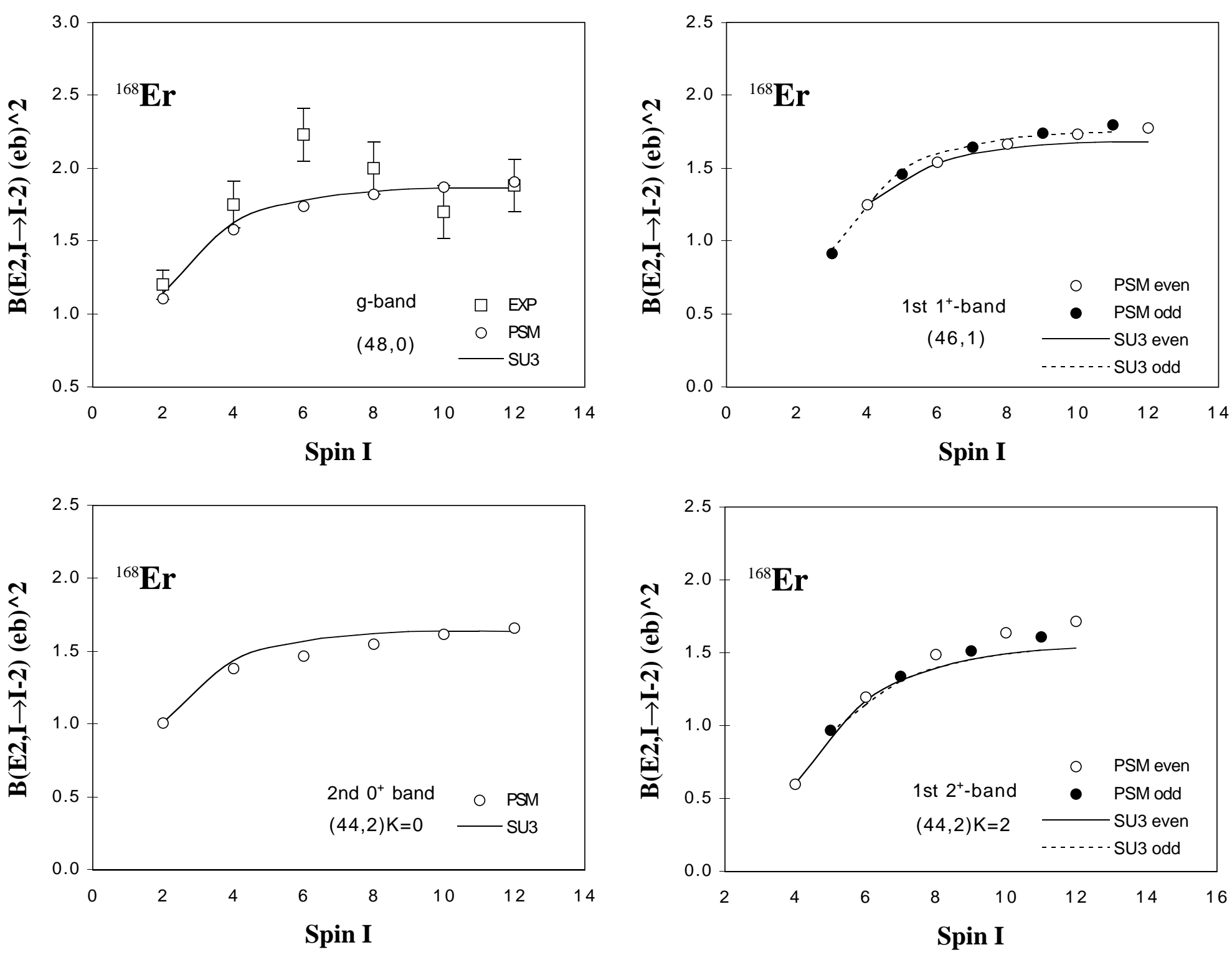

Fig. 3, Sun, Wu, Bhatt and Guidry 


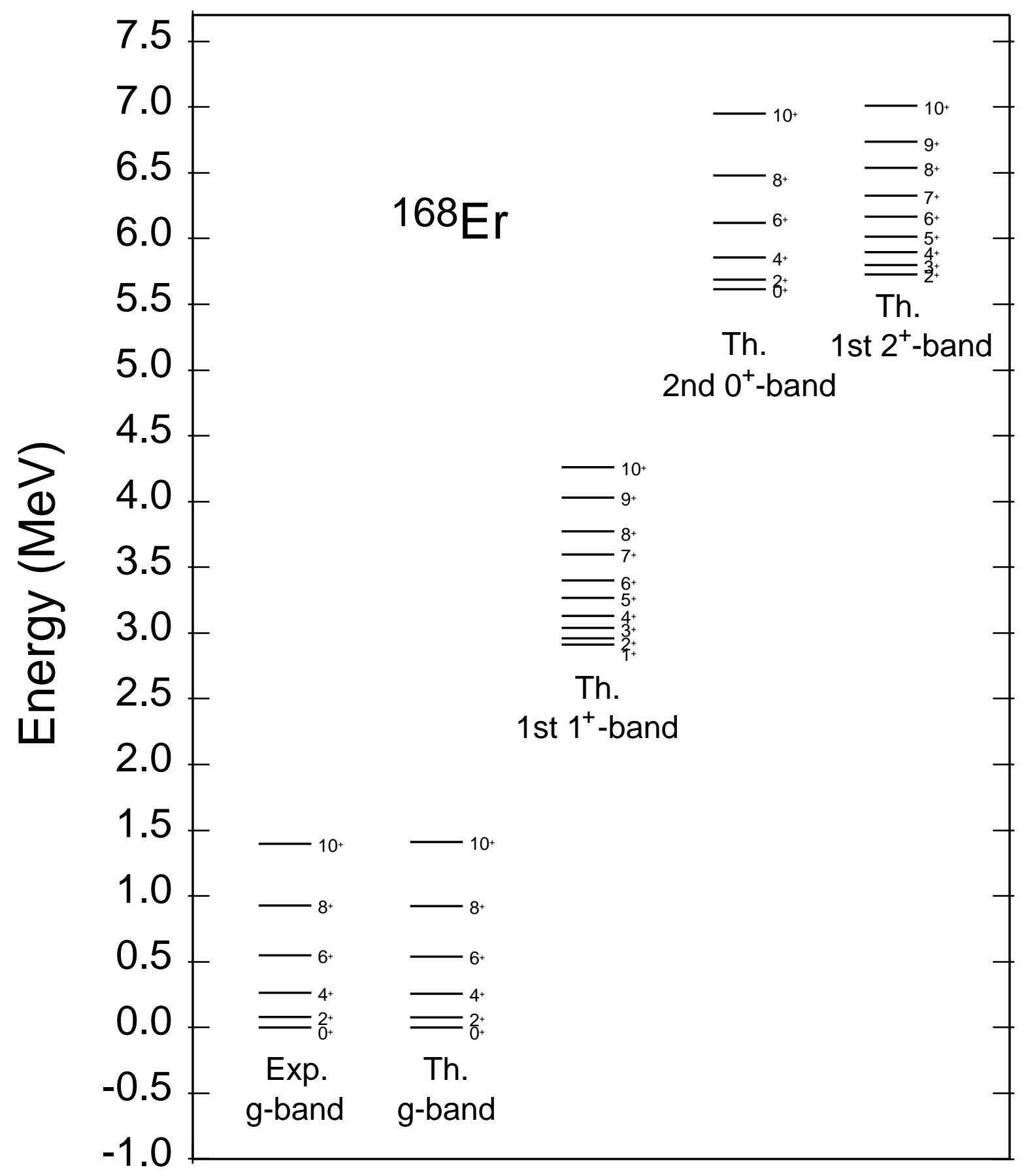

Fig. 4, Sun, Wu, Bhatt and Guidry 